\title{
Soporte circunferencial posterior en fracturas de platillo tibial
}

\author{
Franco L. De Cicco, Jonathan M. Verbner, Mariano O. Abrego, Danilo R. Taype, Guido S. Carabelli, \\ Jorge D. Barla, Carlos F. Sancineto \\ Sector de Trauma Ortopédico, Instituto de Ortopedia y Traumatología “Prof. Dr. Carlos E. Ottolenghi”, Hospital Italiano de Buenos Aires, \\ Ciudad Autónoma de Buenos Aires, Argentina
}

\begin{abstract}
RESUMEN
Las técnicas quirúrgicas que más se utilizan en la actualidad para fracturas de platillo tibial no contemplan correctamente la conminución ósea multiplanar ni la presencia de fragmentos óseos posteriores. Nos hemos enfrentado con esta problemática al tratar a un paciente con fractura de platillo tibial tipo Schatzker VI, en la cual se suplementó la osteosíntesis habitualmente utilizada con una placa horizontal circunferencial posterior. Se logró la consolidación ósea y los resultados funcionales a corto plazo fueron buenos. Del análisis de la bibliografía citada, se concluye en que se han desarrollado varias técnicas de contención posterior de los platillos tibiales, y la osteosíntesis circunferencial es una técnica por considerar.

Palabras clave: Fracturas de tibia; meseta tibial; fijación interna; abordaje posteromedial.

Nivel de Evidencia: IV
\end{abstract}

Posterior Circumferential Support in Tibial Plateau Fractures

\section{ABSTRACT}

Surgical techniques commonly used today for tibial plateau fractures do not properly contemplate multiplanar bone comminution nor the presence of posterior bone fragments. We have faced this problem when treating a patient with a Schatzker VI type fracture, in which the commonly used osteosynthesis, is supplemented with a posterior horizontal circumferential plate. Bone consolidation and good results were achieved in the short term in the case we present. From the analysis of the cited literature, we concluded that several techniques for posterior support of tibial plateau bone comminution have been developed. Among them, circumferential osteosynthesis is a technique to be considered.

Key words: Tibial fractures; tibial plateau; internal fixation; posteromedial approach.

Level of Evidence: IV

\section{INTRODUCCIÓN}

Las fracturas complejas de ambos platillos tibiales (Schatzker V y VI/AO-OTA tipo C) pueden tener distintos patrones. En estos casos, las opciones quirúrgicas varían desde tutores externos hasta la fijación interna con placas y tornillos. Sin embargo, las técnicas más utilizadas no consideran correctamente la conminución multiplanar ni la presencia de fragmentos posteriores. ${ }^{1}$

Nos hemos enfrentado con este problema al tratar a una paciente con fractura tipo Schatzker VI con conminución posterior, a la que decidimos fijar con una placa anterolateral bloqueada de tibia proximal y una placa en " $T$ " asociada a una placa horizontal circunferencial posterior.

El objetivo de este reporte es describir el tratamiento de una paciente con fractura compleja de platillo tibial con conminución posterior y realizar una revisión bibliográfica. Se han evaluado los casos publicados, las técnicas quirúrgicas, la osteosíntesis utilizada, así como los abordajes y las técnicas propuestas.

Recibido el 26-9-2019. Aceptado luego de la evaluación el 19-4-2020 • Dr. FRANCO L. DE CICCO • franco.decicco@ hospitalitaliano.org.ar

https://orcid.org/0000-0001-9844-140X Cómo citar este artículo: De Cicco FL, Verbner JM, Abrego MO, Taype DR, Carabelli GS, Barla JD, Sancineto CF. Soporte circunferencial posterior en fracturas de platillo tibial. Rev Asoc Argent Ortop Traumatol 2021;86(2):219-227. https://doi.org/10.15417/issn. 1852-7434.2021.86.2.1018 


\section{CASO CLÍNICO}

Mujer de 76 años, sin antecedentes clínicos de relevancia, que sufre un accidente de baja energía en la vía pública. Inicialmente, fue atendida en otro centro asistencial donde se le diagnosticó una fractura de platillo tibial de rodilla derecha. Una vez estabilizada, fue derivada a nuestro centro hospitalario con tracción esquelética transcalcánea. La paciente presenta tumefacción, derrame articular y un hematoma medial en la rodilla derecha; no se observan alteraciones neurovasculares.

El par radiológico de rodilla y la tomografía computarizada revelan una fractura de platillo tibial clasificada como Schatzker VI (Figuras 1 y 2). Con la resonancia magnética de rodilla diferida, se diagnostica una lesión de tercer grado asociada del ligamento colateral medial.

La paciente es operada a los cuatro días del accidente por el estado de partes blandas.

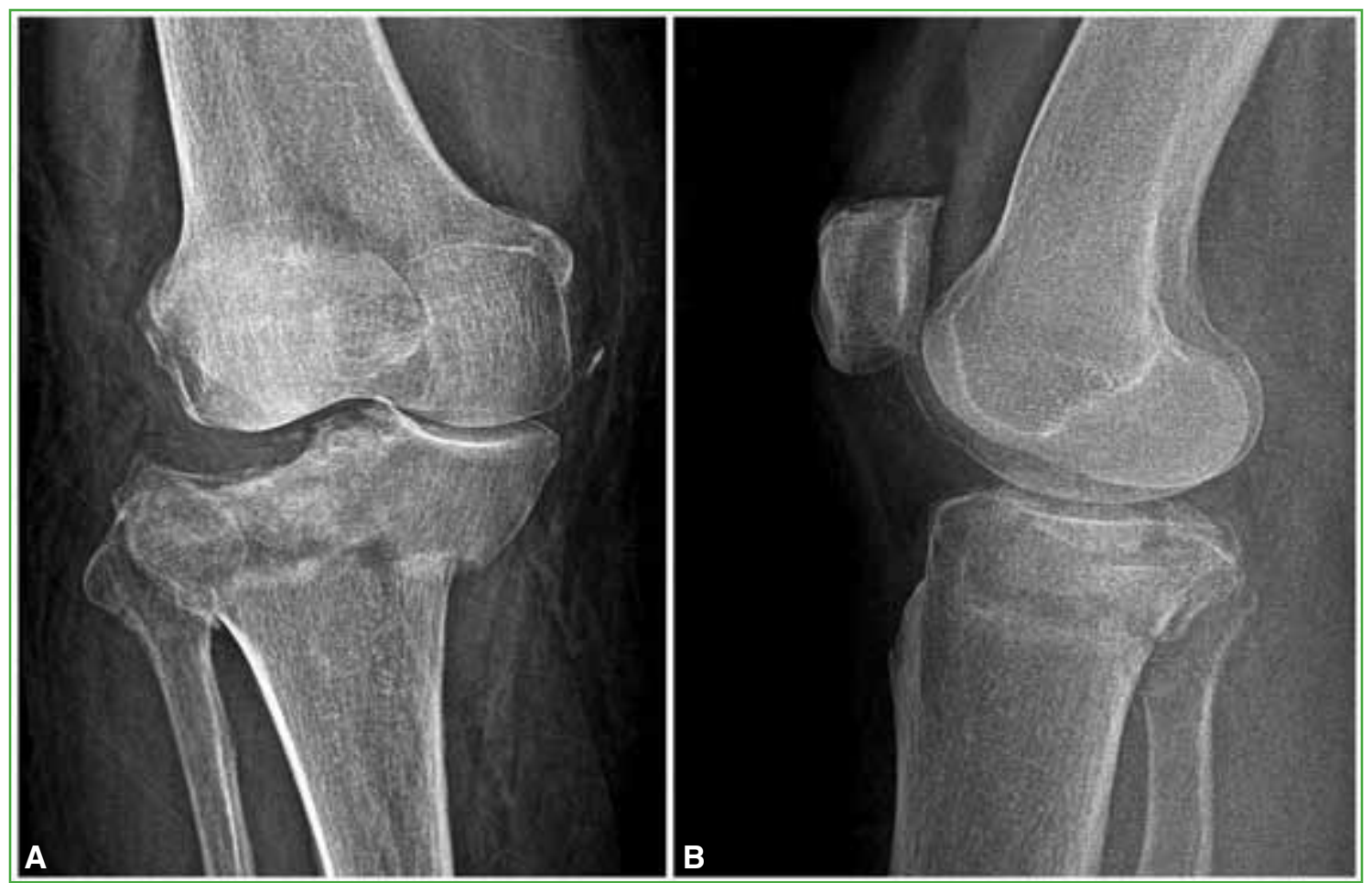

Figura 1. Radiografía inicial de rodilla, de frente (A) y de perfil (B).

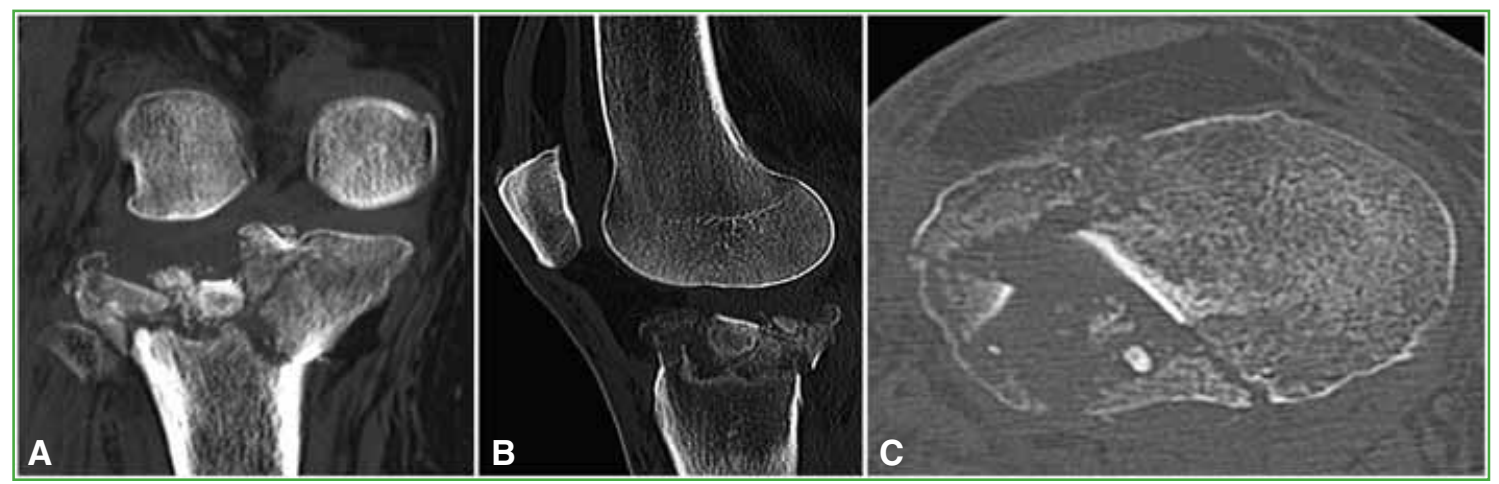

Figura 2. Tomografía computarizada inicial de rodilla. A. Corte coronal posterior. B. Corte sagital. C. Corte axial. 


\section{Técnica quirúrgica}

Con el par radiológico y los cortes de la tomografía, se realiza la planificación preoperatoria. En este caso, la paciente fue derivada con tracción transcalcánea; por ende, no fue necesario retirar el tutor externo. Se coloca a la paciente en decúbito dorsal con realce en la hemipelvis izquierda sobre una mesa radiolúcida. Se procede a una asepsia y antisepsia adecuadas del miembro inferior derecho hasta la cresta ilíaca homolateral inclusive, en el campo quirúrgico y, antes de exanguinar el miembro, se coloca una venda Esmarch hemostática en el muslo.

En primera instancia, se realiza un abordaje posteromedial. Se coloca el miembro en abducción y rotación externa con una ligera flexión de rodilla. El abordaje se extiende desde el epicóndilo femoral medial hasta el borde posterior de la tibia a nivel de la unión metafisodiafisaria. Dicha incisión debe ser recta o ligeramente curvada, se puede prolongar tanto a proximal como a distal, si es necesario. Luego de atravesar el tejido celular subcutáneo, se identifica la fascia del gemelo interno y los tendones de la pars anserina. Se incide en dicho intervalo para tener un mejor acceso posterior. Mediante una disección manual digital se colabora con la separación de los planos musculares y óseos evitando lesión de estructuras nobles con instrumental quirúrgico. Luego se retrae el tendón del músculo semimembranoso hacia proximal y medial; el músculo gemelo interno hacia distal y lateral. Si hay tensión que inhabilite una adecuada visualización, sugerimos desinsertar parcialmente la inserción proximal y medial del músculo sóleo. De esta manera, obtenemos una amplia visualización de la columna posteromedial y un acceso indirecto a la porción posterior del platillo lateral.

Una vez identificada la fractura se efectúa la reducción indirecta mediante la tracción longitudinal del miembro e hiperextensión de la rodilla y luego se estabiliza el fragmento principal transitoriamente con clavijas. A continuación, se procede a la reducción directa con la colocación de una placa posteromedial de 3,5 mm en "T" en función de sostén.

Ante la insuficiencia de esta configuración para contener todos los fragmentos, se decide, entonces, contornear una placa LCP de reconstrucción de 3,5 mm y 8 orificios contorneando el reborde articular del platillo tibial para que cumpla la función de un sostén periarticular. Se logra resolver dicha necesidad mediante una disección roma digital del reborde posterior tibial externo. Se contornea la mitad lateral de la placa emulando el platillo lateral. Una vez deslizada, con la ayuda de dos destornilladores, se completa el contorneado de la mitad medial de la placa logrando así una coaptación circunferencial y se la fija luego con tornillos bloqueados.

Posteriormente se retira el realce y se reposiciona a la paciente en decúbito dorsal estricto. Se coloca un distractor femoral en función de distracción en varo del platillo tibial externo para luego proceder al abordaje anterolateral que se extiende desde el aspecto posterior del cóndilo lateral, dirigiéndose a un punto localizado a dos traveses de dedo hacia lateral de la cresta tibial, distal a la tuberosidad anterior de la tibia. La incisión debe estar centrada entre la cabeza del peroné y el tubérculo de Gerdy. Los dos tercios distales de esta incisión suelen ser suficientes para el abordaje, aunque si es necesario, puede extenderse a proximal. Si se lleva a cabo una técnica mínimamente invasiva, se selecciona el tercio medio de esta incisión. Luego de atravesar el tejido celular subcutáneo, se identifica la banda iliotibial y se incide siguiendo la orientación de sus fibras. Por debajo se encuentra el músculo tibial anterior, que se desinserta en su porción proximal y anterior para darle lugar a la osteosíntesis. Por último, se realiza una capsulotomía transversal distal al menisco lateral para visualizar la reducción articular adquirida. Se reduce la fractura otorgando contención mediante un injerto óseo autólogo tricortical de cresta ilíaca. Se realizan la reducción directa y la estabilización con una placa bloqueada regional de tibia proximal de 5 orificios y 3,5 mm (Figura 3).

Por último, ante la inestabilidad varo-valgo, se reconstruye el ligamento colateral medial con un arpón de 4,5 mm y se corrobora la buena estabilidad clínica y radiológica posterior. Se efectúa un lavado profuso de las heridas con solución fisiológica y se cierra por planos en forma convencional con puntos separados. La tomografía del posoperatorio mostró una adecuada reducción de la fractura (Figura 4).

El protocolo de rehabilitación temprana incluyó ejercicios isométricos activos y marcha en monopedestación con asistencia de un andador de cuatro puntos de apoyo. Por tener una reconstrucción ligamentaria, se mantuvo a la paciente protegida con una férula en extensión de rodilla por 45 días. Luego, comenzó con ejercicios activos y pasivos de rango de movilidad articular y logra una extensión máxima de $20^{\circ}$ y una flexión de $70^{\circ} / 80^{\circ}$ a la semana de retirar la férula. El puntaje de dolor fue 7/10 en la escala analógica visual. A los dos meses y medio, con evidencia radiológica de consolidación ósea, la paciente comenzó con carga parcial progresiva protegida. Los controles evolutivos a los cuatro y seis meses muestran una extensión completa y una flexión de $130^{\circ} / 135^{\circ}$; un puntaje 2 en la escala analógica visual para dolor y un puntaje funcional de 60/90 en el Knee Society Score. Se le otorgó el alta médica con control anual. 


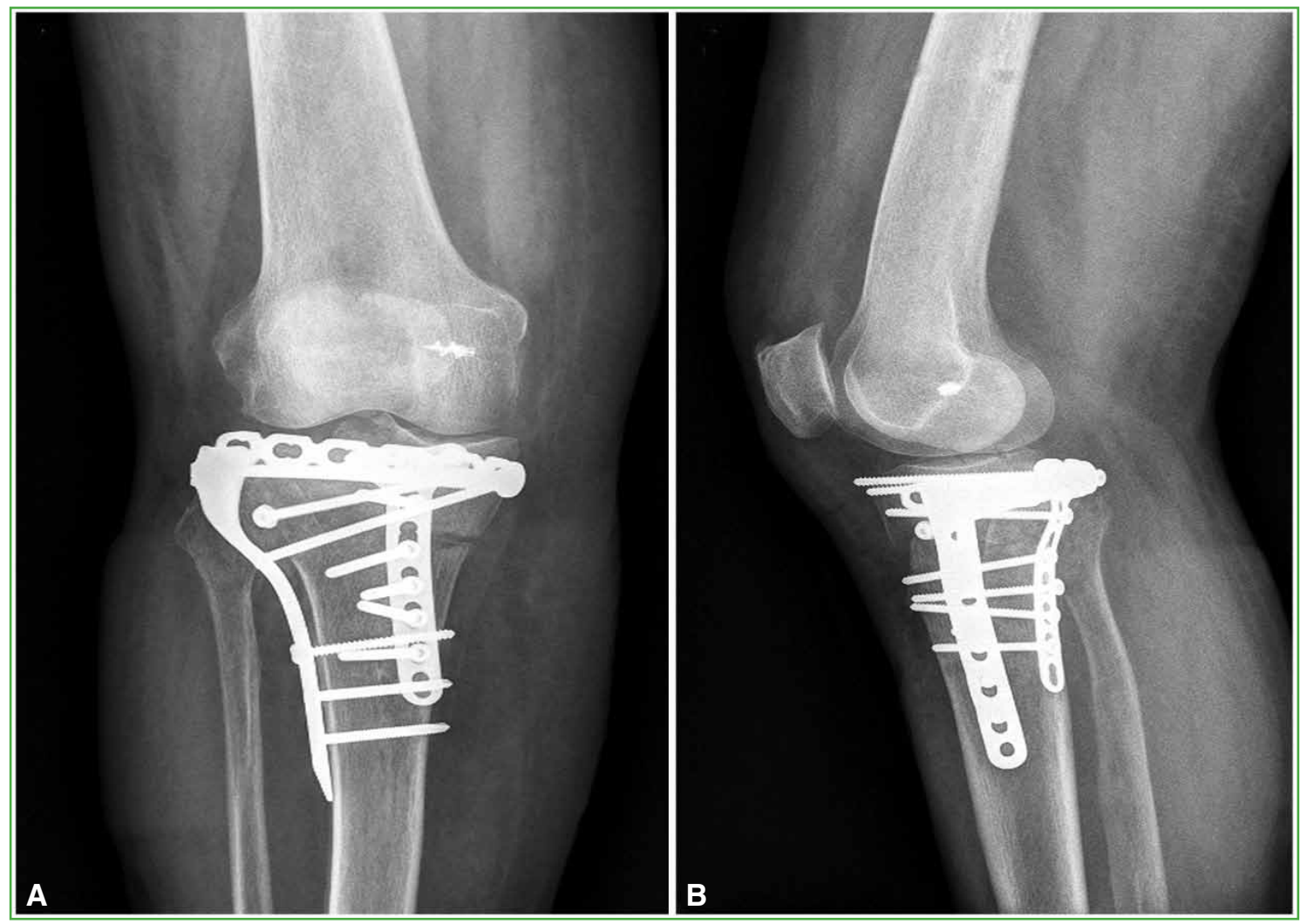

Figura 3. Radiografía posoperatoria de rodilla, de frente (A) y de perfil (B).

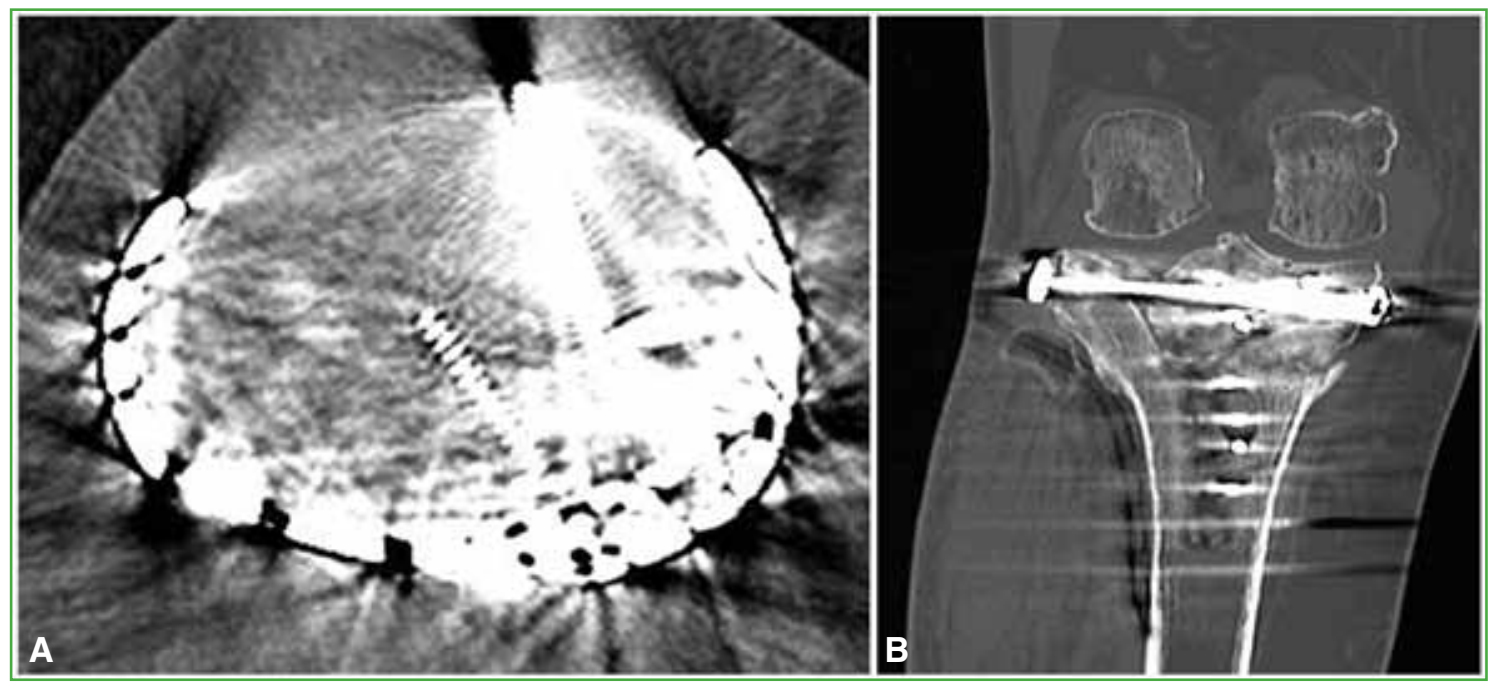

Figura 4. Tomografía computarizada posoperatoria de rodilla. A. Corte axial. B. Corte coronal posterior. 


\section{DISCUSIÓN}

El problema que se presenta en los casos de fracturas de platillo tibial comienza con la comprensión de la fractura y con la decisión del tipo de osteosíntesis por utilizar. Las clasificaciones clásicas más utilizadas de fracturas de platillo tibial se basan solo en proyecciones radiográficas de frente, como lo son las clasificaciones de la $\mathrm{AO} /$ OTA y Schatzker. ${ }^{2-5}$ Esto inclina al cirujano a darle más relevancia a la fijación medial y lateral, descuidando el compromiso posterior. ${ }^{6}$ Sumado a esto, la conminución dificulta aún más la interpretación del patrón fracturario, convirtiendo a estas fracturas posteriores en lesiones que suelen pasar desapercibidas y subestimadas en la planificación quirúrgica.

Se demostró que el plan quirúrgico se modificó en el 59\% de los casos cuando se usaron las imágenes tomográficas. ${ }^{7}$ De esta manera, algunos autores han propuesto que los fragmentos posteromediales son mucho más frecuentes de lo que se publica. ${ }^{8}$ Esto impulsó a autores, como Kahn y cols. ${ }^{9}$ a crear una clasificación tomográfica de platillos tibiales que incluya los fragmentos posteriores. Posteriormente, Luo y cols. ${ }^{6}$ crearon el "concepto de 3 columnas" y Chang y cols. ${ }^{10}$ dividieron en "cuadrantes" a los platillos tibiales utilizando cortes axiales tomográficos. Por último, Chen y cols. ${ }^{5}$ crearon una nueva clasificación de fracturas posteriores de platillo tibial, denominadas "split posterior" ${ }^{11} \mathrm{o}$ "cuña posterior". ${ }^{2}$

Se ha analizado la morfología de los fragmentos posteromediales y posterolaterales, justificando las diferencias de los fragmentos en la asimetría de los cóndilos femorales. ${ }^{5}$ Se ha sugerido que estas fracturas siguen un patrón común, una fractura en el platillo medial, que es cóncavo, suele originar dos fragmentos largos (posteromedial y anteromedial) sin hundimiento. ${ }^{13}$ El fragmento posteromedial suele presentar un trazo cortical en forma de "V". Por otro lado, el platillo externo convexo se fractura y se deprime con un grado de conminución variable.

La bibliografía sobre el tema incluye varios intentos de desarrollar técnicas de contención posterior de los platillos tibiales en los últimos años. La importancia de ello radica en la primordial función de la columna medial en el eje mecánico del miembro. En la actualidad, el tratamiento elegido para fracturas de platillo tibial bicondíleas es el doble abordaje y la fijación con placas (medial y lateral) en función de sostén..$^{10,14,15}$ Sin embargo, esta técnica es insuficiente para fracturas con conminución articular multiplanar, en especial cuando hay un fragmento posterior o un trazo de fractura coronal y cercano a la cortical posterior. ${ }^{1,3,8,16} \mathrm{El}$ uso de modernas placas bloqueadas no logra el soporte necesario para estos fragmentos posteriores. ${ }^{1,3}$ Colocándolas anteriormente, su contorno premoldeado no permitiría el uso de tornillos bloqueados para asegurar aquellos fragmentos posteriores. ${ }^{17}$ Según lo explicado por De Boeck y cols., ${ }^{11}$ utilizando únicamente un abordaje anterior, se podrían colocar tornillos con dirección anteroposterior, pero es dificultoso lograr una correcta reducción de esta forma.

Buscando una solución a esta problemática, varios autores han innovado y descrito nuevas técnicas para la contención de fracturas de compromiso posterior. Sin embargo, en su mayoría, describen técnicas utilizadas en casos con patrones sin conminución y con fragmentos simples posteromediales o posterolaterales.

En 1994, Georgiadis y cols. ${ }^{18}$ publicaron el uso de placas posteriores antideslizamiento para la contención de los fragmentos posteriores. Desde ese entonces, la placa posterior vertical fue la osteosíntesis común para estos patrones y existen numerosas series de casos reportadas (Tabla). En general, estas configuraciones buscan darle sostén a los fragmentos posteriores con la placa posterior y paralelamente utilizar, si es necesario, placas laterales para que sus tornillos mantengan unidos ambos platillos tibiales.

Zeng y cols. ${ }^{19}$ llevaron a cabo un estudio biomecánico de cuatro métodos de fijación de fragmentos posteromediales de platillo tibial: tornillos interfragmentarios anteroposteriores de $6,5 \mathrm{~mm}$, placa LC-DCP anteromedial de $4,5 \mathrm{~mm}$ y 6 orificios, placa bloqueada lateral de 5 orificios de tibia proximal y placa en " $T$ " posterior de 3,5 $\mathrm{mm}$. Simularon 28 fracturas de platillo tibial en hueso sintético y expusieron a estas configuraciones a una carga axial midiendo la fuerza requerida para la falla en cada tipo de construcción. Con este estudio se demostró que una placa posterior en "T" con función de sostén es, desde el punto de vista biomecánico, la configuración más estable in vitro para fragmentos posteromediales.

En 2008, Bermúdez y cols. ${ }^{17}$ describieron la colocación de una placa horizontal para estabilizar fragmentos posteriores de cada platillo tibial. La posición horizontal de la placa permite colocar tornillos en dirección anteroposterior que pasen a través de los fragmentos óseos que se deseen fijar. El moldeado de la placa agrega mayor efecto de sostén sin necesidad de colocar tornillos en la parte posterior de la placa, sino solo en sus extremos para fijarla. Estos autores sugieren usar una placa de reconstrucción de 3,5 mm, debido a su maleabilidad para el moldeado. Si es necesario retirar el implante, la extracción se puede realizar por los abordajes estándares, sin abordajes posteriores, evitando así lesionar elementos neurovasculares posteriores. Como desventaja, esta construcción es insuficiente para la inestabilidad metafisaria posterior. 
Tabla. Revisión bibliográfica

\begin{tabular}{|c|c|c|c|c|c|}
\hline Año & Autor & Estudio & Abordaje & Posición & Tipo de osteosíntesis \\
\hline 1994 & $\begin{array}{l}\text { Georgiadis y } \\
\text { cols. }^{18}\end{array}$ & $\begin{array}{l}\text { Serie de casos } \\
\text { (4 pacientes) }\end{array}$ & Anterolateral y posteromedial & $\begin{array}{l}\text { Decúbito } \\
\text { supino }\end{array}$ & $\begin{array}{l}\text { Placa tercio de tubo contorneada o } \\
\text { placa de sostén en "T" }\end{array}$ \\
\hline 1995 & $\begin{array}{l}\text { De Boeck y } \\
\text { cols. }^{11}\end{array}$ & $\begin{array}{l}\text { Retrospectivo } \\
\text { ( } 7 \text { pacientes) }\end{array}$ & Posteromedial en "S" & $\begin{array}{l}\text { Decúbito } \\
\text { prono }\end{array}$ & Una placa en "T" de sostén \\
\hline 1997 & $\begin{array}{l}\text { Lobenhoffer y } \\
\text { cols. }^{21}\end{array}$ & $\begin{array}{l}\text { Retrospectivo } \\
\text { (168 casos) }\end{array}$ & $\begin{array}{l}\text { Posteromedial y transperoneo } \\
\text { (más osteotomía de peroné) }\end{array}$ & $\begin{array}{l}\text { Decúbito } \\
\text { prono }\end{array}$ & $\begin{array}{l}\text { Tornillos de compresión } \\
\text { interfragmentaria más placa de sostén }\end{array}$ \\
\hline 2005 & $\begin{array}{l}\text { Bhattacharyya } \\
\text { y cols. }{ }^{16}\end{array}$ & $\begin{array}{l}\text { Serie de casos } \\
\text { retrospectiva } \\
\text { (13 pacientes) }\end{array}$ & Posterior en "S" & $\begin{array}{l}\text { Decúbito } \\
\text { prono }\end{array}$ & Una placa en trébol de $3,5 \mathrm{~mm}$ \\
\hline 2005 & $\begin{array}{l}\text { Carlson y } \\
\text { cols. }^{12}\end{array}$ & $\begin{array}{l}\text { Serie de casos } \\
(5 \text { casos })\end{array}$ & Posteromedial y posterolateral & $\begin{array}{l}\text { Decúbito } \\
\text { prono }\end{array}$ & Dos placas de sostén paralelas \\
\hline 2008 & Weil y cols. ${ }^{3}$ & Técnica quirúrgica & Posteromedial y lateral & $\begin{array}{l}\text { Decúbito } \\
\text { supino }\end{array}$ & Una placa posterior antideslizamiento \\
\hline 2008 & $\begin{array}{l}\text { Bermúdez y } \\
\text { cols. }^{17}\end{array}$ & $\begin{array}{l}\text { Reporte de casos } \\
\text { ( } 2 \text { casos) }\end{array}$ & $\begin{array}{l}\text { Anterolateral extendido y } \\
\text { posteromedial }\end{array}$ & $\begin{array}{l}\text { Decúbito } \\
\text { prono }\end{array}$ & Una o dos placas horizontales de sostén \\
\hline 2009 & $\begin{array}{l}\text { Brunner y } \\
\text { cols. }^{22}\end{array}$ & $\begin{array}{l}\text { Serie de casos } \\
(5 \text { casos })\end{array}$ & Posterior directo en "S" & $\begin{array}{l}\text { Decúbito } \\
\text { prono }\end{array}$ & $\begin{array}{l}\text { Placa de } 3,5 \mathrm{~mm} \text { con función de sostén } \\
\text { y antideslizamiento }\end{array}$ \\
\hline 2010 & Yu y cols. ${ }^{23}$ & $\begin{array}{l}\text { Técnica quirúrgica } \\
\text { Prospectivo ( } 82 \text { pacientes) }\end{array}$ & $\begin{array}{l}\text { Anterolateral más osteotomía } \\
\text { de peroné }\end{array}$ & $\begin{array}{l}\text { Decúbito } \\
\text { supino }\end{array}$ & Placa de sostén en "L" \\
\hline 2010 & Frosch y cols. ${ }^{24}$ & Técnica quirúrgica & Posterolateral modificado & $\begin{array}{l}\text { Decúbito } \\
\text { lateral }\end{array}$ & Una placa en "L" de $3,5 \mathrm{~mm}$ \\
\hline 2010 & $\begin{array}{l}\text { Lugones y } \\
\text { cols. }^{25}\end{array}$ & $\begin{array}{l}\text { Técnica quirúrgica } \\
\text { (15 pacientes) }\end{array}$ & Posteromedial y lateral & $\begin{array}{l}\text { Decúbito } \\
\text { prono }\end{array}$ & Una placa en $\mathrm{T}$ de $4,5 \mathrm{~mm}$ \\
\hline 2010 & Luo y cols. ${ }^{6}$ & $\begin{array}{l}\text { Cohorte prospectiva } \\
\text { ( } 29 \text { casos) }\end{array}$ & $\begin{array}{l}\text { Posterior en "L" invertida } \\
\text { (para fractura de columna } \\
\text { media o columna posterior) y } \\
\text { anterolateral }\end{array}$ & $\begin{array}{l}\text { Decúbito } \\
\text { supino } \\
\text { flotante }\end{array}$ & Una placa de sostén \\
\hline 2012 & Chang y cols. ${ }^{10}$ & $\begin{array}{l}\text { Prospectivo } \\
\text { (12 pacientes) }\end{array}$ & Posteromedial y anterolateral & $\begin{array}{l}\text { Decúbito } \\
\text { supino } \\
\text { flotante }\end{array}$ & $\begin{array}{l}\text { Triple plaqueado ( } 1 \text { placa lateral + } \\
1 \text { placa medial }+1 \text { placa posterior } \\
\text { coronal antideslizamiento) }\end{array}$ \\
\hline 2013 & He y cols. ${ }^{4}$ & $\begin{array}{l}\text { Serie de casos }(8 \\
\text { pacientes })\end{array}$ & Posterior en "L" invertida & $\begin{array}{l}\text { Decúbito } \\
\text { prono }\end{array}$ & $\begin{array}{l}\text { Una placa de sostén de } 3,5 \mathrm{~mm} \\
\text { LC-DCP o } 3,5 \mathrm{~mm} \text { en "T" }\end{array}$ \\
\hline 2014 & Aly y cols. ${ }^{20}$ & $\begin{array}{l}\text { Técnica quirúrgica } \\
\text { Prospectivo ( } 29 \text { pacientes) }\end{array}$ & Posteromedial y lateral & $\begin{array}{l}\text { Decúbito } \\
\text { supino }\end{array}$ & $\begin{array}{l}\text { Placa más cerclaje } \\
\text { Banda de tensión }\end{array}$ \\
\hline 2014 & Chang y cols. ${ }^{13}$ & $\begin{array}{l}\text { Técnica quirúrgica } \\
\text { Prospectivo } \\
\text { (16 pacientes) }\end{array}$ & Posteromedial y anterolateral & $\begin{array}{l}\text { Decúbito } \\
\text { supino } \\
\text { flotante }\end{array}$ & Placas de sostén \\
\hline 2015 & Chen y cols. ${ }^{5}$ & Prospectivo (39 pacientes) & $\begin{array}{l}\text { Posteromediales o } \\
\text { posterolaterales en "L" } \\
\text { invertida }\end{array}$ & $\begin{array}{l}\text { Decúbito } \\
\text { prono }\end{array}$ & Placas de sostén en "T" \\
\hline 2015 & $\begin{array}{l}\text { Robledo- } \\
\text { Herrera y cols. }^{2}\end{array}$ & $\begin{array}{l}\text { Observacional } \\
\text { ( } 7 \text { pacientes) }\end{array}$ & Posteromedial & $\begin{array}{l}\text { Decúbito } \\
\text { supino }\end{array}$ & $\begin{array}{l}\text { Placas en "T" de } 3,5 \mathrm{~mm} \text { y } 4,5 \mathrm{~mm} \text { / } \\
\text { placas de reconstrucción/placa en palo } \\
\text { de hockey }\end{array}$ \\
\hline 2016 & Garner y cols. ${ }^{26}$ & $\begin{array}{l}\text { Técnica quirúrgica } \\
\text { Descriptivo }\end{array}$ & $\begin{array}{l}\text { Descripción comparativa de } \\
5 \text { abordajes para el acceso a } \\
\text { fracturas posterolaterales }\end{array}$ & $\begin{array}{l}\text { Según el } \\
\text { abordaje }\end{array}$ & - \\
\hline 2016 & Hake y cols. ${ }^{27}$ & $\begin{array}{l}\text { Técnica quirúrgica } \\
\text { Descriptivo }\end{array}$ & Posteromedial de Lobenhoffer & $\begin{array}{l}\text { Decúbito } \\
\text { prono }\end{array}$ & Placa antideslizamiento \\
\hline 2016 & $\begin{array}{l}\text { Giordano y } \\
\text { cols. }{ }^{1}\end{array}$ & Reporte de caso & Lateral más posteromedial & $\begin{array}{l}\text { Decúbito } \\
\text { supino }\end{array}$ & Placa horizontal de sostén \\
\hline
\end{tabular}


En 2014, Aly y cols..$^{20}$ evaluaron el tratamiento de las fracturas de ambos platillos tibiales desplazadas con cerclaje circunferencial al platillo combinado con una placa con tornillos. La técnica consiste en un abordaje posteromedial y luego la colocación de una placa anterolateral y una lazada de alambre circunferencial a la parte proximal de la tibia. Para facilitar la colocación del alambre, sugieren el uso de una guía específica utilizada en cirugía de plástica de ligamento cruzado anterior. Esta configuración permite realizar cierta compresión centrípeta de los fragmentos gracias a la torsión del alambre.

En 2016, Giordano y cols. ${ }^{1}$ describieron un caso en el que se utilizó una placa tercio de tubo premoldeada colocada de forma horizontal para contener el borde posterior de los platillos tibiales, denominaron a esta técnica como "placa aro" (hoop plate). Efectuaron un abordaje lateral con osteotomía de la cabeza del peroné para lograr la reducción de los fragmentos anterolaterales y posterolaterales bajo visión directa. La placa se coloca desde lateral hacia medial y, para facilitar su colocación, sugieren utilizar un elevador de Cobb para liberar el recorrido que hará la placa antes de colocarla. Los autores aconsejan realizar esta maniobra en flexión de rodilla para evitar lesionar el paquete vasculonervioso. Ya colocada la placa, se la ajusta utilizando un clamp de puntas que comprima a través de los orificios de los extremos de la placa, logrando así la compresión interfragmentaria.

Giordano y cols. sugieren esta técnica para fracturas articulares de platillo tibial con impactación del borde posterior y de la pared cortical. El empleo de una placa tercio de tubo de bajo perfil, moldeable permite la combinación con otras placas, si es necesario.

Como ventaja, las placas horizontales permiten dar contención circunferencial y mantener unidos ambos platillos. También, gracias al uso de tornillos, se puede realizar una compresión interfragmentaria. Si hay fragmentos tan pequeños que no toleren la penetración de un tornillo, se puede lograr la compresión interfragmentaria mediante el pretensado de la placa.

Como desventajas, la extracción de las placas horizontales posteriores es más difícil comparada con la de las placas laterales. También es incompetente para estabilizar la carilla articular con la metáfisis, por lo que requieren una segunda placa que cumple esta función.

Las técnicas habituales para el tratamiento de fracturas complejas de platillo tibial no tienen en cuenta la presencia de fragmentos conminutos en el reborde posterior. Como sugieren Giordano y cols., la contención de los fragmentos corticales y articulares posteriores requiere un tipo de osteosíntesis que tenga una función de sostén centrípeto estabilizando la fractura en el plano axial, ya sea con lazadas de alambre o con placas horizontales moldeadas.

\section{CONSIDERACIONES FINALES}

Las fracturas con conminución posterior son patrones complejos de abordar quirúrgicamente. El uso de una placa moldeada, colocada en forma horizontal desde un abordaje posteromedial permite lograr una contención y un sostén adecuados de los fragmentos posteriores. Se remeda con mayor integridad la anatomía normal del platillo. Contribuye a la fijación del componente posteromedial, principal responsable de contribuir y preservar un eje mecánico adecuado a la rodilla. Constituye una técnica aplicable que podría brindar resultados posoperatorios funcionales similares a los de las configuraciones tradicionales.

Conflicto de intereses: Los autores no declaran conflictos de intereses.

ORCID de J. M. Verbner: https://orcid.org/0000-0001-7040-2097 ORCID de M. O. Abrego: https://orcid.org/0000-0001-9783-7373 ORCID de D. R. Taype: https://orcid.org/0000-0001-8293-9879
ORCID de G. S. Carabelli: https://orcid. org/0000-0002-7049-0688 ORCID de J. D. Barla: https://orcid.org/0000-0001-9233-5278 ORCID de C. F. Sancineto: hitps://orcid.org/0000-0002-5190-4312 


\section{BIBLIOGRAFÍA}

1. Giordano V, Schatzker J, Kfuri M. The "hoop" plate for posterior bicondylar shear tibial plateau fractures: description of a new surgical technique. J Knee Surg 2016;30(6):509-13. https://doi.org/10.1055/s-0036-1593366

2. Robledo-Herrera O, Diego-Ball D, Oliva-Ramirez S. [Posteromedial approach and plating for a tibial plateau fracture with a posterior fragment]. Acta Ortop Mex 2015;29(2):69-76. Disponible en: https://www.medigraphic.com/pdfs/ortope/or-2015/or152b.pdf

3. Weil YA, Gardner MJ, Boraiah S, Helfet DL, Lorich DG. Posteromedial supine approach for reduction and fixation of medial and bicondylar tibial plateau fractures. J Orthop Trauma 2008;22(5):357-62. https://doi.org/10.1097/BOT.0b013e318168c72e

4. He X, Ye P, Hu Y, Huang L, Zhang F, Liu G, et al. A posterior inverted L-shaped approach for the treatment of posterior bicondylar tibial plateau fractures. Arch Orthop Trauma Surg 2013;133(1):23-8. https://doi.org/10.1007/s00402-012-1632-2

5. Chen H-W, Chen C-Q, Yi X-H. Posterior tibial plateau fracture: a new treatment-oriented classification and surgical management. Int J Clin Exp Med 2015;15;8(1):472-9. PMID: 25785019

6. Luo C-F, Sun H, Zhang B, Zeng B-F. Three-column fixation for complex tibial plateau fractures. J Orthop Trauma 2010;24(11):683-92. https://doi.org/10.1097/BOT.0b013e3181d436f3

7. Wicky S, Blaser PF, Blanc CH, Leyvraz PF, Schnyder P, Meuli RA. Comparison between standard radiography and spiral CT with 3D reconstruction in the evaluation, classification and management of tibial plateau fractures. Eur Radiol 2000;10(8):1227-32. https://doi.org/10.1007/s003300000326

8. Barei DP, O'Mara TJ, Taitsman LA, Dunbar RP, Nork SE. Frequency and fracture morphology of the posteromedial fragment in bicondylar tibial plateau fracture patterns. J Orthop Trauma 2008;22(3):176-82. https://doi.org/10.1097/BOT.0b013e318169ef08

9. Khan RM, Khan SH, Ahmad AJ, Umar M. Tibial plateau fractures. A new classification scheme. Clin Orthop Relat Res 2000; (375):231-42. PMID: 10853174

10. Chang S-M, Wang X, Zhou J-Q, Huang Y-G, Zhu X-Z. Posterior coronal plating of bicondylar tibial plateau fractures through posteromedial and anterolateral approaches in a healthy floating supine position. Orthopedics 2012;35(7):583-8. https://doi.org/10.3928/01477447-20120621-03

11. De Boeck H, Opdecam P. Posteromedial tibial plateau fractures. Operative treatment by posterior approach. Clin Orthop Relat Res 1995;(320):125-8. PMID: 7586815

12. Carlson DA. Posterior bicondylar tibial plateau fractures. J Orthop Trauma 2005;19(2):73-8. https://doi.org/10.1097/00005131-200502000-00001

13. Chang S-M, Hu S-J, Zhang Y-Q, Yao M-W, Ma Z, Wang X, et al. A surgical protocol for bicondylar four-quadrant tibial plateau fractures. Int Orthop 2014;38(12):2559-64. https://doi.org/10.1007/s00264-014-2487-7

14. Krieg JC. Proximal tibial fractures: current treatment, results, and problems. Injury 2003;34(Suppl 1):A2-10. https://doi.org/10.1016/s0020-1383(03)00252-3

15. Ricci WM, Rudzki JR, Borrelli J. Treatment of complex proximal tibia fractures with the less invasive skeletal stabilization system. J Orthop Trauma 2004;18(8):521-7. https://doi.org/10.1097/00005131-200409000-00007

16. Bhattacharyya T, McCarty LP, Harris MB, Morrison SM, Wixted JJ, Vrahas MS, et al. The posterior shearing tibial plateau fracture: treatment and results via a posterior approach. J Orthop Trauma 2005;19(5):305-10. PMID: 15891538

17. Bermúdez CA, Ziran BH, Barrette-Grischow M-K. Use of horizontal rafting plates for posterior elements of complex tibial plateau fractures: description and case reports. J Trauma 2008;65(5):1162-7. https://doi.org/10.1097/01.ta.0000222943.45563.b5

18. Georgiadis GM. Combined anterior and posterior approaches for complex tibial plateau fractures. J Bone Joint Surg Br 1994;76(2):285-9. PMID: 8113294

19. Zeng Z-M, Luo C-F, Putnis S, Zeng B-F. Biomechanical analysis of posteromedial tibial plateau split fracture fixation. Knee 2011;18(1):51-4. https://doi.org/10.1016/j.knee.2010.01.006

20. Aly T, Hafez K, Amin O. Treatment of tibial plateau fractures using cerclage-wiring with surface flat plating technique: short term results. Research 2014;1:725. https://doi.org/10.13070/rs.en.1.725

21. Lobenhoffer P, Gerich T, Bertram T, Lattermann C, Pohlemann T, Tscheme H. [Particular posteromedial and posterolateral approaches for the treatment of tibial head fractures]. Unfallchirurg 1997;100(12):957-67. https://doi.org/10.1007/s001130050218 
22. Brunner A, Honigmann P, Horisberger M, Babst R. Open reduction and fixation of medial Moore type II fractures of the tibial plateau by a direct dorsal approach. Arch Orthop Trauma Surg 2009;129(9):1233-8. https://doi.org/10.1007/s00402-009-0841-9

23. Yu B, Han K, Zhan C, Zhang C, Ma H, Su J. Fibular head osteotomy: a new approach for the treatment of lateral or posterolateral tibial plateau fractures. Knee 2010;17(5):313-8. https://doi.org/10.1016/j.knee.2010.01.002

24. Frosch K-H, Balcarek P, Walde T, Stürmer KM. [A modified posterolateral approach for the treatment of tibial plateau fractures]. Oper Orthop Traumatol 2010;22(1):107-19. https://doi.org/10.1007/s00064-010-3008-0

25. Lugones A. Abordaje posteromedial para la reducción y estabilización del componente posteromedial en fracturas del platillo tibial. Rev Asoc Argent Ortop Traumatol 2010;75 (2):144-50. Disponible en: http://www.aaot.org.ar/revista/2010/n2/Rev_AsocArgentOrtopTraumatol_2010_75_144-150_Lugones.pdf

26. Garner MR, Warner SJ, Lorich DG. Surgical approaches to posterolateral tibial plateau fractures. J Knee Surg 2016; 29(1):12-20. https://doi.org/10.1055/s-0035-1564731

27. Hake ME, Goulet JA. Open reduction and internal fixation of the posteromedial tibial plateau via the Lobenhoffer approach. J Orthop Trauma 2016;30(Suppl 2):S35-6. https://doi.org/10.1097/BOT.0000000000000582 